\title{
Universiteit
}

Leiden

The Netherlands

\section{Rate Dependence and Role of Disorder in Linearly Sheared Two- Dimensional Foams}

Katgert, G.; Möbius, M.E.; Hecke, M.L. van

\section{Citation}

Katgert, G., Möbius, M. E., \& Hecke, M. L. van. (2008). Rate Dependence and Role of Disorder in Linearly Sheared Two-Dimensional Foams. Physical Review Letters, 101(5), 058301. doi:10.1103/PhysRevLett.101.058301

Version: $\quad$ Publisher's Version

License: $\quad$ Leiden University Non-exclusive license

Downloaded from: https://hdl.handle.net/1887/71405

Note: To cite this publication please use the final published version (if applicable). 


\title{
Rate Dependence and Role of Disorder in Linearly Sheared Two-Dimensional Foams
}

\author{
Gijs Katgert, * Matthias E. Möbius, and Martin van Hecke \\ Kamerlingh Onnes Lab, Universiteit Leiden, Postbus 9504, 2300 RA Leiden, The Netherlands
}

(Received 26 November 2007; published 28 July 2008)

\begin{abstract}
The shear flow of two-dimensional foams is probed as a function of shear rate and disorder. Disordered, bidisperse foams exhibit strongly shear rate dependent velocity profiles. This behavior is captured quantitatively in a simple model based on the balance of the time-averaged drag forces in the system, which are found to exhibit power-law scaling with the foam velocity and strain rate. Disorder makes the scaling of the bulk drag forces different from that of the local interbubble drag forces, which we evidence by rheometrical measurements. In monodisperse, ordered foams, rate independent velocity profiles are found, which lends further credibility to this picture.
\end{abstract}

DOI: 10.1103/PhysRevLett.101.058301

Similar to other disordered materials such as (colloidal) suspensions, granular media, and emulsions, foams, which are dispersions of densely packed gas bubbles in liquid, exhibit a nontrivial rheology [1-7]. When left unperturbed, foams jam into a metastable state corresponding to a local minimum of the surface energy, where surface tension provides the restoring force underlying their elastic response for small strains [6-8]. Under a continuous driving force, foam bubbles overcome these local minima and the foam starts to flow, and the viscous dissipation that arises near the thin fluid films that surround the gas bubbles becomes important [6]. It is a daunting task to obtain the rheology of foams by coarse graining the bubble-bubble interactions $[3,4,9,10]$. Already for a single bubble sliding past a solid wall, Bretherton showed that the drag force scales nonlinearly with the bubble velocity $[4,11,12]$, and by analogy one would expect the drag forces arising between sliding bubbles to be nonlinear also. In addition, at the multibubble scale, foam flows can be disordered and intermittent $[3,8,9]$. Foams share this combination of nonlinear interactions and complex flows with other disordered media. However, bubble interactions are probably simpler than those of frictional grains, and are similar to those of the soft spheres without static friction that have been studied extensively in the context of jamming [13-15], making foams eminently suited for fundamental studies of the flow of disordered media. In this Letter, we will address the role of disorder for foam flows, by experiments on the rheology of foams both at the coarse grained and at the bubble level. To probe and visualize foam flows, a number of experiments have been conducted recently in quasi-two-dimensional geometries. Here the foam flow is driven by moving sidewalls, and the soap bubbles either form a bubble raft where they freely float on the fluid phase [16], are sandwiched by two glass plates in a Hele-Shaw cell [17], or are trapped between the fluid phase and a top plate $[18,19]$. The presence of such a top plate leads to shear banding of the flow [19]. This can be understood from the additional drag forces exerted on the bubbles flowing under the top plate, which will be balanced by gradients in the bulk stresses of the material.
PACS numbers: 47.57.Bc, 83.50.Rp, 83.80.Iz

A model based on the balance of drag forces which captures the observed shear banding qualitatively was recently introduced by Janiaud et al. [10]. For simplicity, it was assumed that the drag forces exerted by the top plate scale linearly with bubble velocity, and that the bulk stress varies linearly with strain rate. These linear laws lead to rate independent flows [10].

Here we experimentally probe the flow of disordered 2D foams which are trapped between the fluid phase and a top plate. We find that the flow depends crucially on the applied strain rate $\dot{\gamma}_{a}$ : disordered, bidisperse foams exhibit rate dependent flow profiles, which become increasingly shear banded for large $\dot{\gamma}_{a}$.

These findings are captured in a model in which the time-averaged drag forces between bubble and top plate, $\bar{F}_{b w}$, and between neighboring bubbles, $\bar{F}_{b b}$, are balanced. While the continuum limit of our model is similar in spirit to the model of Janiaud, the crucial new ingredient is nonlinear scaling laws for the wall drag and the bulk stress - these nonlinear scalings are essential for capturing the observed rate dependence.

We establish the precise scaling forms of the averaged drag forces in disordered foams by varying the applied shear rate over three orders in magnitude and fitting the data to our model, and confirm these scalings by independent rheological measurements. We furthermore perform rheometrical measurements on ordered lanes of bubbles, which reflect the viscous drag force between individual bubbles. Surprisingly, the averaged drag forces in the disordered foam scales differently from the local drag forces between individual bubbles, and in our range of parameters, the averaged forces are much larger than expected from naively scaling up the local drag forces. In contrast, for monodisperse, ordered foams, the local drag forces, averaged drag forces, and top-plate drag all scale similarly, causing rate independent flows [19]. We attribute the modification of the drag forces to the disordered and nonaffine motion of the bubbles in the bidisperse foam $[3,9,13,14]$.

Setup. - A bidisperse (50:50 number ratio) bubble monolayer is produced by flowing nitrogen through two 
syringe needles immersed at fixed depth in a soapy solution consisting of 5\% volume fraction Dawn dish washing liquid and $15 \%$ glycerol in demineralized water (viscosity $\eta=1.8 \pm 0.1 \mathrm{mPas}$ and surface tension $\sigma=28 \pm$ $1 \mathrm{mN} / \mathrm{m}$ ). The resulting bubbles of $1.8 \pm 0.1$ and $2.7 \pm$ $0.1 \mathrm{~mm}$ diameter are gently mixed to produce a disordered bidisperse monolayer and are covered with a glass plate (see Fig. 1). The weighted average bubble diameter $\langle d\rangle$ is $2.25 \mathrm{~mm}$.

Two parallel acrylic glass wheels of $195 \mathrm{~mm}$ radius and $9 \mathrm{~mm}$ thickness are partially immersed in the liquid through $10 \mathrm{~mm}$ wide slits in the top plate such that they are in contact with the foam over a length of $230 \mathrm{~mm}$, while having an adjustable gap distance $W$ ranging from 50 to $100 \mathrm{~mm}$ (Fig. 1). The wheels have a roughness of order $3 \mathrm{~mm}$ at the contact line due to etched grooves, like the spokes on a bicycle wheel, to ensure no slip boundaries for the bubbles, and are counterrotated by two microstepper motors. The bubbles contact the top plate, which is completely wetted by the soap solution, and we fix the liquid fraction of the foam by keeping the distance between glass plate and liquid surface fixed at $2.25 \pm 0.01 \mathrm{~mm}$. Coalescence, segregation, and coarsening as well as the drag force between bubbles and fluid phase are negligible.

The average velocity $v(y)$ in the $\hat{\mathbf{x}}$ direction is obtained from both particle tracking and particle image velocimetry techniques. Since the time-resolved flow is strongly disordered and intermittent [Fig. 2(a)], we average over time and over $x$, where we restrict the $x$ range to a central region of length $60 \mathrm{~mm}$ [Fig. 1(a)] where recirculation is negligible $\left(\left\langle v_{y}\right\rangle=0\right)$.

Rate dependent flows. - We measured the flow profiles $v$ for gap width $W$ equal to 5,7 , and $9 \mathrm{~cm}$, and driving velocities $v_{0}=0.026,0.083,0.26,0.83,2.6$, and $8.3 \mathrm{~mm} / \mathrm{s}$. In Fig. 2 we show a few examples of these. The main observation is that the velocity profiles strongly vary with the driving velocity $v_{0}$, and become increasingly shear banded for large $v_{0}$.

Drag force balance model. - The flow profiles and the scaling forms of the drag forces are connected by a simple model in which the average drag forces are balanced. As illustrated in Fig. 2(c), we divide the foam in lanes of width $\langle d\rangle$ and balance the time-averaged top plate drag per bubble $\bar{F}_{b w}^{i}$ with the time-averaged viscous drag per bubble due to the lane to the left $\left(\bar{F}_{b b}^{i}\right)$ and right $\left(\bar{F}_{b b}^{i+1}\right)$ :

$$
\bar{F}_{b b}^{i+1}-\bar{F}_{b w}^{i}-\bar{F}_{b b}^{i}=0 .
$$

Even though the instantaneous velocities fluctuate strongly, we assume that we can express the average drag forces in terms of the average velocities $v^{i}$. We nondimensionalize velocities according to the definition of the capillary number $(\mathrm{Ca}:=\eta v / \sigma)$, and propose

$$
\begin{gathered}
\bar{F}_{b w}^{i}=f_{b w}\left(\eta v^{i} / \sigma\right)^{\alpha}, \\
\bar{F}_{b b}^{i}=f_{Y}+f_{b b}\left[(\eta / \sigma)\left(v^{i}-v^{i-1}\right)\right]^{\beta},
\end{gathered}
$$
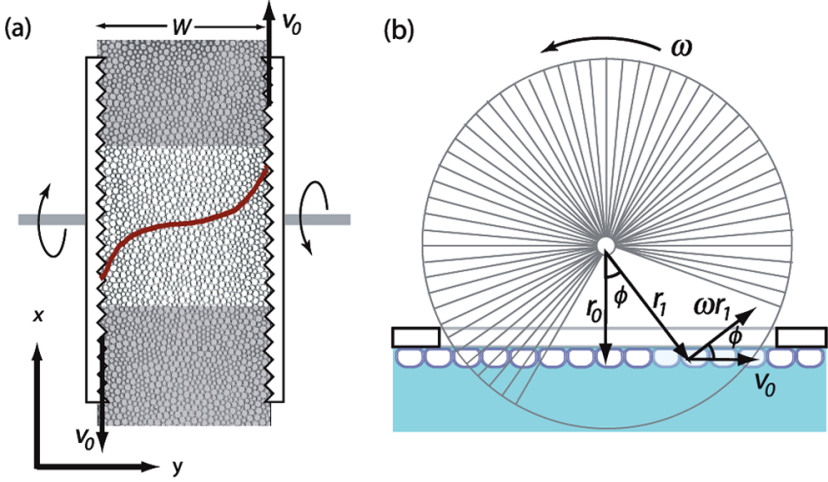

FIG. 1 (color online). (a) Schematic top view of the experimental setup, showing how two counterrotating wheels partially immersed in the fluid and spaced by a gap $W$ shear the foam. Data are taken in the highlighted area and a typical flow profile is indicated. (b) Side view showing the layer of bubbles trapped below the top plate and the grooved shearing wheels. $v_{0}$ is the $x$ component of the wheels' angular velocity, and is equal to $\omega r_{0}$ over the contact line, since $v_{0}=\omega r_{1} \cos \phi=\omega \frac{r_{0}}{\cos \phi} \times$ $\cos \phi=\omega r_{0}$. The applied strain rate $\dot{\gamma}_{a}$ equals $2 v_{0} / W$.

$$
\bar{F}_{b b}^{i+1}=f_{Y}+f_{b b}\left[(\eta / \sigma)\left(v^{i+1}-v^{i}\right)\right]^{\beta} .
$$

The expression for $\bar{F}_{b w}$ is essentially the result for a single bubble sliding past a solid wall, for which Bretherton showed that the drag force $F_{b w}$ scales nonlinearly with the capillary number $[4,11,12,20] . f_{b w}$ is a constant with dimensions of force of order $\sigma r_{c}$, where $r_{c}$ is the radius of the bubble-wall contact [20]. The power-law index $\alpha$ depends on the surfactant. Dawn dish washing liquid has a low surface shear modulus [21], for which $\alpha=2 / 3$ [4] [see Fig. 3(a)].

For $\bar{F}_{b b}$ we conjecture a Herschel-Bulkley-type expression, which combines a finite threshold $f_{Y}$ with a powerlaw dissipative term. The crucial exponent $\beta$ will be determined from the flow profiles and rheology below.

Inserting these expressions into Eq. (1) and defining $k=$ $f_{b w} / f_{b b}$ we arrive at

$$
k\left(\frac{\eta v^{i}}{\sigma}\right)^{\alpha}=\left(\frac{\eta}{\sigma}\right)^{\beta}\left[\left(v^{i+1}-v^{i}\right)^{\beta}-\left(v^{i}-v^{i-1}\right)^{\beta}\right],
$$

where it should be noted that the yield threshold $f_{Y}$ drops out of the equations of motion - we keep it here to remain consistent with our rheological measurements (see Fig. 3).

Model versus experimental flow profiles. - To compare our model [Eq. (5)] to the 18 experimental flow profiles obtained for three widths and six driving velocities, we need to determine the two-dimensionless parameters $\beta$ and $k$. To avoid being affected by edge effects near the shearing wheels, we focus on the part of the data where $|v|<$ $(3 / 4) v_{0}$, and solve Eq. (5) by numerically integrating from where $v=0$ to the $y$ value for which $v=(3 / 4) v_{0}$. For fixed $\beta$ and $k$ we can thus compare the experimental data and model prediction. 

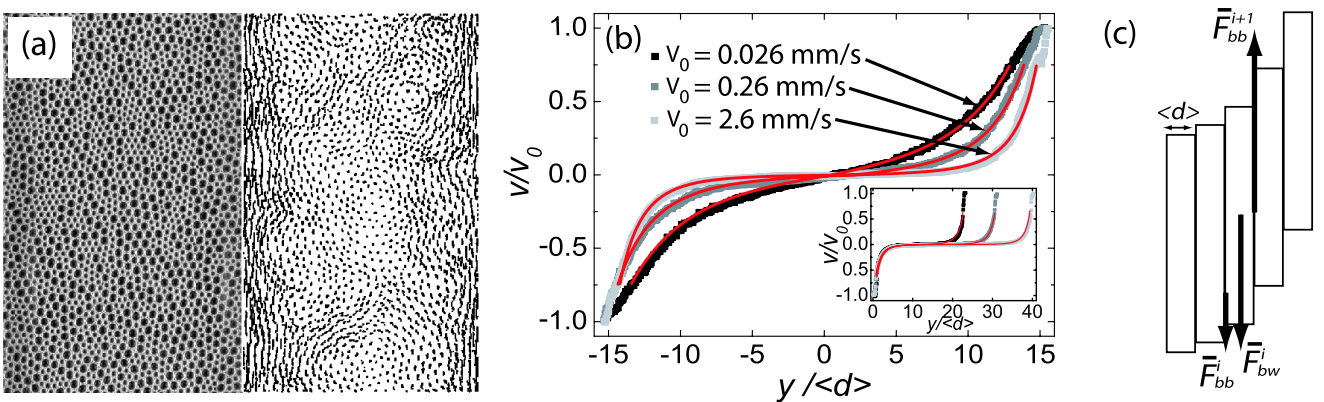

FIG. 2 (color online). (a) Experimental image of bidisperse foam (left) and corresponding bubble tracks (right). Note the swirling motion. (b) Rescaled velocity profiles $v / v_{0}$ for $W=7 \mathrm{~cm}$ and $v_{0}$ as indicated, compared to profiles obtained from our model Eq. (5) with $\alpha=2 / 3, \beta=0.36$, and $k=3.75$ [thin solid (red) lines]. Inset: $v / v_{0}$ for $v_{0}=8.3 \mathrm{~mm} / \mathrm{s}$ and $W$ equal to 5,7 , and $9 \mathrm{~cm}-$ for convenience, we chose the origin at the left boundary here. (c) Illustration of the model defined by Eq. (5).

To determine $\beta$ and $k$, we require that all profiles are fit well for the same values of these fitting parameters. When $\beta$ is not chosen optimally, we find that $k$ systematically varies with $v_{0}$, but for $\beta=0.36 \pm 0.05$, this systematic variation is minimized. We find that for $\alpha=0.67, \beta=$ 0.36 , and $k=3.75 \pm 0.5$, all 18 data sets can be fit excellently by our model (Fig. 2) [22].

Constitutive relation. - By taking the continuum limit we can rewrite our model Eq. (5) as the balance between a 1D body force and gradient of the 1D stress $\tau$ :

$$
\begin{aligned}
& f_{b w}\left(\frac{\eta v}{\sigma}\right)^{2 / 3}\langle d\rangle^{-1}=\frac{\partial \tau}{\partial y}, \\
& \tau=\tau_{Y}+f_{b b}\left(\frac{\eta\langle d\rangle \dot{\gamma}}{\sigma}\right)^{0.36},
\end{aligned}
$$

where $\tau_{Y}$ is an undetermined yield stress. This yields the constitutive equation for a Herschel-Bulkley fluid, and the value $\beta=0.36$ is remarkably close to recent results for $3 \mathrm{D}$ bulk rheology of emulsions and foams $[2,4]$.
Rheological determination of $\alpha, \beta$, and $k$. - The force laws that underlie our model can be probed directly by rheological measurements, and we have measured the bubble-wall and interbubble forces with an Anton Paar MCR-501 rheometer [see Figs. 3(a) and 3(b)]. We find that $\alpha=0.67 \pm 0.02$, thus confirming that $F_{b w}$ is given by Bretherton's law for mobile surfactants. The measured value of the exponent $\beta, 0.40 \pm 0.02$, is within error bars to what we found by simply fitting the model to the flow profiles. We extract from the rheological measurements an estimate for the ratio $k=f_{b w} / f_{b b} \approx 2.5 \pm 0.5$. This is close to the value $k=3.75 \pm 0.5$ estimated from the flow profiles [23].

Rheological determination of $\beta$ at the bubble scale.-To see if the effective interbubble drag force corresponds trivially to the drag forces at the bubble scale, we have probed this force by narrowing the gap width of our Couette geometry so that two pinned and perfectly ordered lanes of bubbles slide past each other, and find that here
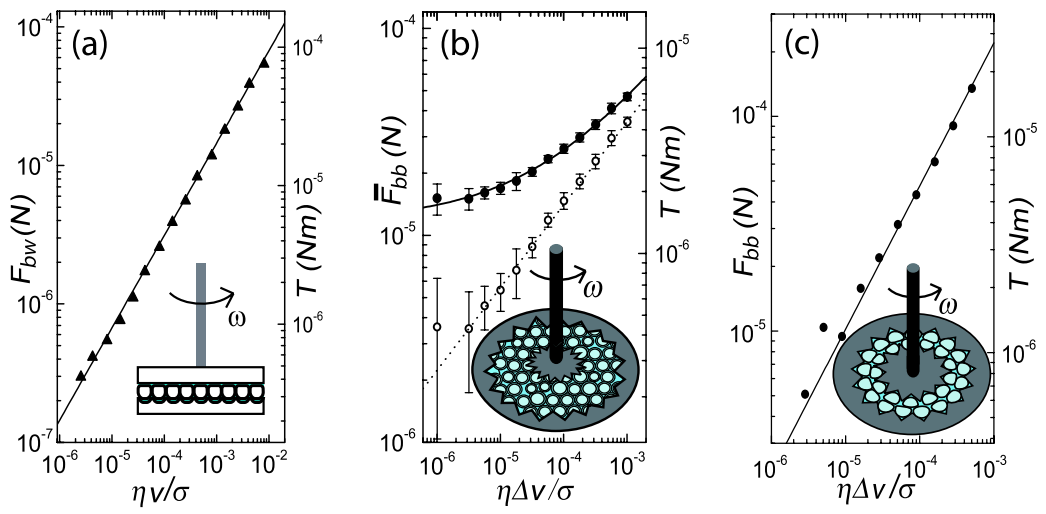

FIG. 3 (color online). (a) $F_{b w}$ is deduced by trapping a monolayer of bubbles between a rough bottom and a smooth top plate of radius $20 \mathrm{~mm}$ (inset). From the power-law scaling of torque $T$ we deduce the drag force per bubble as a function of Ca [4] and find that $F_{b w}=f_{b w} \mathrm{Ca}^{0.67 \pm 0.02}$, with $f_{b w} \approx 1.5 \pm 0.1 \times 10^{-3} \mathrm{~N}$. (b) $\bar{F}_{b b}$ is deduced from the time-averaged torque exerted on our bidisperse foam as a function of $\Delta \mathrm{Ca}(\equiv \eta \Delta v / \sigma)$ (filled circles). The foam is sheared in a Couette cell of inner radius $1.25 \mathrm{~cm}$, outer radius $2.5 \mathrm{~cm}$ (hence a gap of 5 bubble diameters) without a top plate (inset). We obtain $\bar{F}_{b b}=f_{Y}+f_{b b}(\Delta \mathrm{Ca})^{\beta}$, with the yield threshold $f_{Y} \approx 1.2(5) \times 10^{-5} \mathrm{~N}, f_{b b} \approx 5.6(9) \times 10^{-4} \mathrm{~N}$, and $\beta=0.40(2)$ (solid line). Open circles are the same data with the yield torque obtained from the fit subtracted, which are well fit by a pure power law with exponent 0.4 (dashed line). (c) Drag force between two pinned and ordered bubble lanes in a Taylor-Couette geometry. The black line indicates power-law scaling with exponent $2 / 3$. 

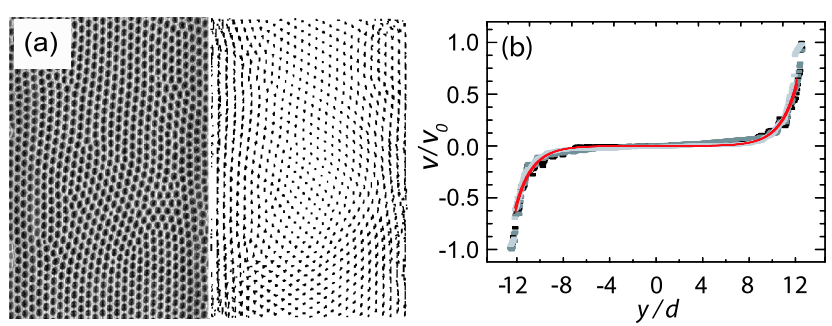

FIG. 4 (color online). (a) Experimental image of monodisperse foam (left) and bubble tracks (right). Note the order in the foam and the absence of swirly motion in the tracks. (b) Velocity profiles for a monodisperse foam $(d=2.7 \mathrm{~mm})$ at $7 \mathrm{~cm}$ gap, for $0.083 \mathrm{~mm} / \mathrm{s}$ (black curve), $0.26 \mathrm{~mm} / \mathrm{s}$ (dark gray curve), and $0.83 \mathrm{~mm} / \mathrm{s}$ (light gray curve). Thin solid (red) lines are fits to the model with both $\alpha$ and $\beta$ equal to $2 / 3$, and $k=0.3$.

$\bar{F}_{b b} \sim(\Delta v)^{2 / 3}$ [Fig. 3(c)] [24]. We believe that this result reflects the actual viscous drag force between individual bubbles sliding past one other, which implies that the average drag forces in a bidisperse, disordered foam deviate in a highly nontrivial way from the drag forces at the bubble level.

Discussion. - The drag forces exerted on the bubbles by the top plate, which at first sight might be seen as obscuring the bulk rheology of the foam, enable us to back out the effective interbubble drag forces and constitutive relation of foams from the average velocity profiles. By comparing these results with rheometrical measurements, we note a remarkable difference between the scaling of the drag forces at the bubble level and the bulk level: we find $F_{b b} \sim$ $(\Delta v)^{2 / 3}$ at the bubble level and $\bar{F}_{b b} \sim(\Delta v)^{0.36}$ at the bulk level.

One might understand this anomalous scaling as follows: The degree of disorder does not affect the drag forces at the bubble scale, but it does modify the bubble motion. For disordered foams, the bubbles exhibit nonaffine and irregular motion-hence they "rub" their neighboring bubbles much more than when they would flow orderly, and consequently, the averaged viscous dissipation is enhanced over what could naively be expected from the local drag forces [9]. This picture is corroborated by recent simulations on the bubble model [3], where one recovers this "renormalization" of the drag force exponent [25,26] and rate-dependent flow profiles [26]. To further illustrate our picture, we linearly shear a monodisperse foam $(d=$ $2.7 \mathrm{~mm}$ ) and recover rate independent profiles (see Fig. 4). By tracking we confirm the absence of significant disordered bubble motion in this case. Our model only yields rate independent profiles if $\alpha=\beta$ so that $\beta=2 / 3$, which implies that without disorder the connection between local and bulk drag forces is trivial. This also solves the conundrum of why Wang et al. [19] found rate independent flow profiles, as their foams are essentially monodisperse.

In conclusion, polydisperse, disordered foams exhibit rate dependent flows due to anomalous scaling of the averaged drag forces $\bar{F}_{b b}$. We suggest that anomalous scaling of bulk properties caused by nonaffine motion at the particle scale may be a general feature of disordered systems close to jamming [3,9,13-15].

The authors wish to thank Jeroen Mesman for technical assistance. G. K. and M.M. acknowledge support from physics foundation FOM, and M. v. H. acknowledges support from NWO/VIDI.

*katgert@ physics.leidenuniv.nl

[1] T. G. Mason, J. Bibette, and D.A. Weitz, J. Colloid Interface Sci. 179, 439 (1996); W. Losert et al., Phys. Rev. Lett. 85, 1428 (2000); P. Coussot et al., ibid. 88, 218301 (2002); R. Besseling et al., ibid. 99, 028301 (2007).

[2] L. Bécu, S. Manneville, and A. Collin, Phys. Rev. Lett. 96, 138302 (2006).

[3] D. J. Durian, Phys. Rev. Lett. 75, 4780 (1995); Phys. Rev. E 55, 1739 (1997).

[4] N. D. Denkov et al., Colloids Surf. A 263, 129 (2005).

[5] C. Gilbreth, S. Sullivan, and M. Dennin, Phys. Rev. E 74, 051406 (2006).

[6] A. M. Kraynik, Annu. Rev. Fluid Mech. 20, 325 (1988).

[7] R. Höhler and S. Cohen-Addad, J. Phys. Condens. Matter 17, R1041 (2005).

[8] D. Weaire and S. Hutzler, The Physics of Foams (Clarendon, Oxford, 1999).

[9] A. J. Liu et al., Phys. Rev. Lett. 76, 3017 (1996); S. A. Langer and A. J. Liu, J. Phys. Chem. B 101, 8667 (1997); S. Tewari et al., Phys. Rev. E 60, 4385 (1999).

[10] E. Janiaud, S. Hutzler, and D. Weaire, Phys. Rev. Lett. 97, 038302 (2006).

[11] F. P. Bretherton, J. Fluid Mech. 10, 166 (1961).

[12] E. Terriac, J. Etrillard, and I. Cantat, Europhys. Lett. 74, 909 (2006).

[13] C. S. O'Hern et al., Phys. Rev. E 68, 011306 (2003).

[14] W. G. Ellenbroek et al., Phys. Rev. Lett. 97, 258001 (2006).

[15] P. Olsson and S. Teitel, Phys. Rev. Lett. 99, 178001 (2007).

[16] J. Lauridsen, M. Twardos, and M. Dennin, Phys. Rev. Lett. 89, 098303 (2002).

[17] G. Debrégeas, H. Tabuteau, and J. M. di Meglio, Phys. Rev. Lett. 87, 178305 (2001).

[18] B. Dollet, M. Aubouy, and F. Graner, Phys. Rev. Lett. 95, 168303 (2005).

[19] Y. Wang, K. Krishan, and M. Dennin, Phys. Rev. E 73, 031401 (2006).

[20] P. Aussillous and D. Quéré, Europhys. Lett. 59, 370 (2002).

[21] S. A. Koehler, S. Hilgenfeldt, and H. A. Stone, Phys. Rev. Lett. 82, 4232 (1999).

[22] Edge effects extend further into the bulk for smaller $v_{0}$, and consistent with this, the worst fits are for small channel width $W=50 \mathrm{~mm}$ and the lowest values of $v_{0}$.

[23] G. Katgert, M.E. Möbius, and M. van Hecke (to be published).

[24] D. A. Reinelt and A. M. Kraynik, J. Colloid Interface Sci. 132, 491 (1989).

[25] V. J. Langlois, S. Hutzler, and D. Weaire [Phys. Rev. E (to be published)].

[26] J. Remmers (private communications). 